\title{
Mineração
}

\section{Condutividade hidráulica saturada de um rejeito de minério de ferro}

\author{
(Saturated hydraulic conductivity of a iron tailing)
}

\author{
Alexandre Gonçalves Santos \\ Companhia Siderúrgica Nacional, Congonhas, Minas Gerais, Brasil \\ E-mail: alexandre.santos@csn.com.br
}

Luís Fernando Martins Ribeiro

Universidade de Brasília, Brasília, Distrito Federal, Brasil

E-mail:Imartins@unb.br

\section{Resumo}

Atualmente o crescente aumento do volume de rejeitos gerados pelo aproveitamento de minerais com teor de ferro cada vez mais baixos tem motivado as mineradoras a adotar, como solução principal para disposição desses rejeitos, a técnica do aterro hidráulico aliada ao método de alteamento a montante. Essa metodologia pressupõe o aparecimento de incertezas relacionadas ao processo de deposição na praia e ao próprio rejeito, que é lançado de forma aleatória e sem controle das variáveis de descarga que influenciam nesse processo. Assim, podem formar-se regiões na barragem com alta concentração de ferro, o que tende a influenciar as propriedades geotécnicas desse material. Com base no relatado anteriormente, o objetivo desse trabalho constituiu em estudar o comportamento do rejeito, em função da porcentagem de ferro e sílica nele presente. Também foi verificada influência do rejeito na condutividade hidráulica saturada, através de ensaios de laboratório e ensaios de campo.

Palavras-chave: Barragens de rejeitos, teor de ferro, condutividade hidráulica.

\begin{abstract}
Nowadays the increased tailing volume, generated by the utilzation of minerals with low iron, has been motivating mining companies to adopt the technique hydraulic fill structures with upstream method as the main solution for tailings disposal. This methodology is influenced by the appearance of uncertainties related to the deposition process for the beach and to the tailing that is launched randomly and without control of the discharge variables that influence this process. As such, high iron concentration areas are formed in the dam, tending to influence the geotechnical properties of this material. Thus, the objective was to study tailing behavior in function of the percentage of iron and silica present and to verify their influence in the saturated hydraulic conductivity of this tailing through laboratory and field tests.
\end{abstract}

Keywords: Tailings dams, iron content and hydraulic conductivity. 


\section{Introdução}

A utilização da técnica do aterro hidráulico em sistemas de disposição de rejeitos tem sido o método mais atraente para as mineradoras, pois proporciona uma redução de custos de construção pela facilidade na execução de barragens e por viabilizar a utilização do rejeito nestas estruturas de contenção. Entretanto problemas construtivos e de segurança podem ocorrer, principalmente devido à falta de controle das variáveis de descarga durante o lançamento e das características do rejeito.

Na técnica do aterro hidráulico, o material é lançado hidraulicamente e o transporte pode ser feito por bombeamento ou gravidade. Normalmente, este lançamento é realizado com a utilização de hidrociclones ou canhões. No caso de utilização de canhões, o processo de separação ocorre na própria praia, em função da velocidade de descarga, concentração e características mineralógicas do rejeito, enquanto que, nos hidrociclones uma classificação granulométrica é feita antes do lançamento.

No caso de lançamento com canhões, algumas variáveis como vazão e concentração da mistura lançada conduzem para um alto grau de heterogeneidade do material depositado. Esse tipo de seleção é função do processo de segregação ocorrido na praia e conduz à formação de zonas com propriedades geotécnicas distintas, principalmente no que se refere ao valor da densidade e granulometria do depósito formado.

Com base na heterogeneidade desses depósitos, o objetivo principal desse trabalho é estudar o comportamento do rejeito em função da porcentagem de ferro presente no rejeito e verificar sua influência nas propriedades geotécnicas. Assim foi realizado um programa de ensaios de campo e laboratórios, avaliando a influência do teor de ferro no valor da densidade do depósito, bem como seu efeito na condutividade hidráulica saturada do rejeito.

\section{Aterros hidráulicos aplicados a barragens de rejeitos}

A execução do aterro hidráulico pelo Método de Montante constitui a opção mais econômica e de maior facilidade executiva. Contudo, pela simplicidade executiva, tende a apresentar maiores problemas de controle de qualidade e segurança. A segregação granulométrica gerada no processo de disposição é uma característica importante nos aterros hidráulicos e tende a provocar uma seleção granulométrica em função do tamanho, da forma e da densidade das partículas, gerando uma variabilidade estrutural e alterando, significativamente, as características de resistência, deformabilidade e permeabilidade.

No caso do rejeito de minério de ferro, o processo de segregação gera, em determinadas regiões da barragem de rejeito, zonas de alta concentração de partículas de ferro (Ribeiro, 2000). Dessa forma, o efeito da densidade das partículas de ferro condiciona o processo de seleção através de um padrão de segregação condicionado ao peso das partículas de ferro presentes nesse rejeito. Nesse contexto, verifica-se a importância de analisar a densidade obtida no campo e, considerando o rejeito de minério de ferro, observa-se que esse parâmetro está diretamente influenciado pela massa especifica dos grãos. Assim, uma tendência de análise das propriedades geotécnicas dos rejeitos estaria presente na avaliação das características dos rejeitos. Essa conclusão tem como base o teor de ferro e sua influência no comportamento das barragens.

Na execução do programa experimental de laboratório, foram utilizadas amostras obtidas para diferentes faixas de variação do teor de ferro em função de um processo de deposição a partir de ensaios de simulação de deposição hidráulica (ESDH) desenvolvido por Ribeiro (2000). É importante ressaltar que esse equipamento possibilita simular um processo de deposição hidráulica semelhante ao que ocorre no campo. Adicionalmente ao programa experimental de la- boratório, foram realizadas análises do valor da condutividade hidráulica na Mina do Complexo das Águas Claras (CVRD) em Rio Piracicaba. Os ensaios realizados foram de infiltração, utilizando o permeâmetro de Guelph, e foram realizados em diferentes regiões da barragem e tendo como referência o ponto de lançamento de rejeitos.

\section{Caracterização geotécnica dos rejeitos}

Os estudos realizados compreenderam ensaios de laboratório, considerando amostras de um rejeito basicamente arenoso proveniente do processo de beneficiamento do minério de ferro da Mina do Complexo de Água Limpa (CVRD), situada no município de Rio Piracicaba a $140 \mathrm{Km}$ de Belo Horizonte.

É importante ressaltar que as amostras obtidas no ESDH foram coletadas em diferentes pontos ao longo do canal de deposição tendo como referência o ponto de lançamento. A denominação adotada, para identificar essas amostras, foi feita em função desse parâmetro. A amostra MA8-000 foi coletada imediatamente abaixo do ponto de lançamento. As amostras MA8-040, MA8-080 e MA8120 foram coletadas a 40, 80 e $120 \mathrm{~cm}$ do ponto de lançamento.

Para os ensaios de campo, adotouse o mesmo procedimento de avaliar as propriedades do rejeito em função da distância do ponto de lançamento. Assim a campanha de campo foi realizada através da definição de pontos localizados ao longo da praia de rejeitos. Dessa forma, foi realizada uma marcação em campo de uma grande malha quadrangular de 30 x 40 m. As seções longitudinais foram espaçadas em 10 metros a partir da crista e as seções transversais distantes 10 metros entre si (Figura 1).

A adoção dessas seções objetivou avaliar o processo de deposição hidráulica no campo durante formação do aterro hidráulico, bem como os efeitos da mudança da posição dos pontos de lançamento ao longo da crista da barragem. 
Alexandre Gonçalves Santos et al.

Devido o rejeito ser constituído, basicamente, de partículas de ferro e quartzo, uma pequena variação no teor de ferro pode causar modificações significativas no valor da massa específica dos grãos do rejeito. $\mathrm{O}$ valor da densidade dos grãos dos materiais estudados variou entre 2,89 e 4,41.

Os valores do teor de ferro das amostras foram obtidos utilizando a correlação encontrada por Espósito (2000) para esse mesmo rejeito a partir da relação entre a massa específica dos grãos e a porcentagem de ferro. Espósito (2000) obteve a evidência de uma relação linear crescente ao avaliar as relações entre o teor de ferro e a massa específica dos grãos de pontos amostrados em diferentes regiões na pilha de rejeitos no campo.

A Tabela 1 apresenta os resultados dos ensaios de caracterização realizados nas amostras obtidas para determinação da densidade dos grãos e seus respectivos valores de teores de ferro obtidos através da equação encontrada por Espósito (2000):

$\rho_{\mathrm{s}}=0,025 \% \mathrm{Fe}+2,6$

Onde: $\rho_{\mathrm{s}}$ é a massa específica dos grãos e \%Fe é o teor de ferro das amostras.

Considerando a mesma metodologia de avaliação para as amostras obtidas no campo, a Tabela 2 apresenta resultados dos ensaios realizados para determinação da densidade dos grãos, dos índices de vazios máximo e mínimo e dos respectivos teores de ferro em cada ponto ao longo da praia de rejeitos (Figura 1).

\section{Caracterização da condutividade hidráulica in situ}

O programa experimental de campo contou com a realização conjunta de ensaios para determinação condutividade hidráulica e ensaios para a determinação do índice de vazios de campo. Para avaliação da condutividade hidráulica foram realizados ensaios de infiltração utilizan-

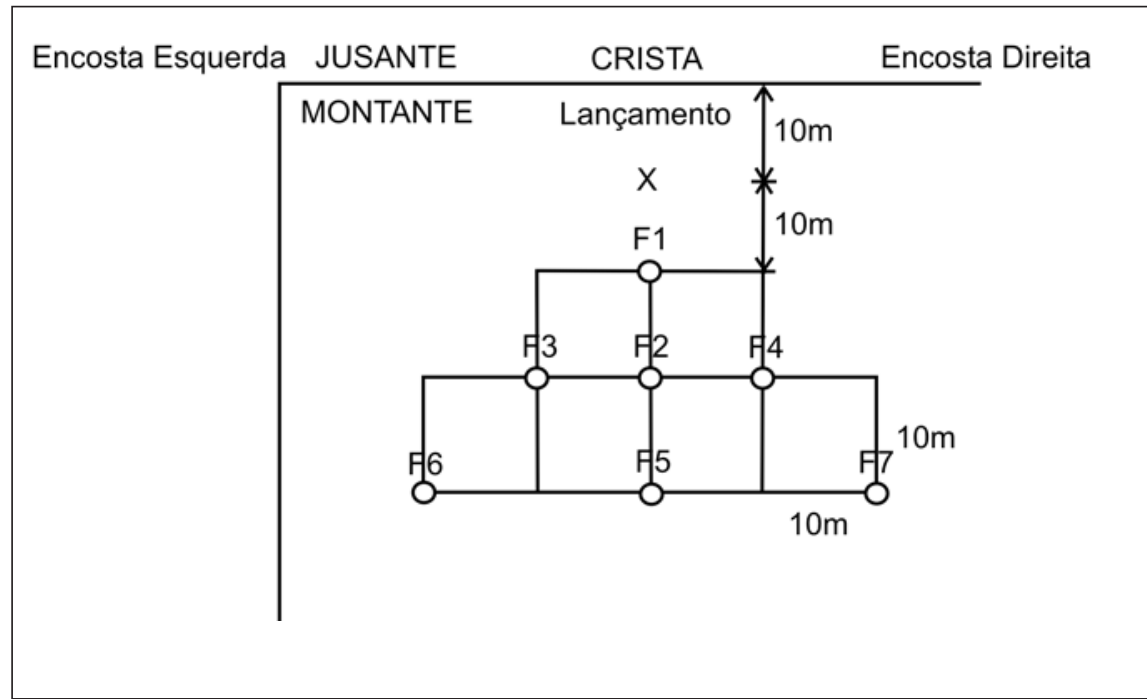

Figura 1 - Seções de investigação ao longo da praia de rejeitos.

Tabela 1 - Parâmetros geotécnicos dos rejeitos amostrados através do ESDH.

\begin{tabular}{c|c|c|c|c}
\hline Amostra & $\mathbf{G}_{\mathbf{s}}$ & $\mathbf{F e} \%$ & $\mathbf{e}_{\min }$ & $\mathbf{e}_{\max }$ \\
\hline MA8-000 & 4,41 & 72,40 & 0,65 & 1,04 \\
\hline MA8-040 & 3,17 & 22,72 & 0,63 & 0,95 \\
\hline MA8-080 & 3,04 & 17,60 & 0,60 & 0,90 \\
\hline MA8-120 & 2,89 & 11,44 & 0,58 & 0,90 \\
\hline
\end{tabular}

Tabela 2 - Parâmetros geotécnicos dos rejeitos amostrados no campo.

\begin{tabular}{c|c|c|c|c}
\hline Amostra & $\mathbf{G}_{\mathbf{s}}$ & $\mathbf{F e} \%$ & $\mathbf{e}_{\min }$ & $\mathbf{e}_{\max }$ \\
\hline Furo 01 & 3,14 & 21,64 & 0,58 & 1,00 \\
\hline Furo 02 & 3,16 & 22,48 & 0,59 & 1,00 \\
\hline Furo 03 & 3,14 & 21,44 & 0,56 & 0,96 \\
\hline Furo 04 & 3,01 & 16,56 & 0,51 & 0,94 \\
\hline Furo 05 & 3,19 & 23,72 & 0,56 & 0,97 \\
\hline Furo 06 & 2,95 & 13,96 & 0,52 & 0,88 \\
\hline Furo 07 & 2,95 & 13,88 & 0,53 & 0,94 \\
\hline
\end{tabular}

do o permeâmetro de Guelph. A análise da densidade in situ foi feita através do método do cilindro biselado. A campanha foi realizada considerando as mesmas seções propostas na malha quadrangular definida na praia de rejeitos e já apresentada na Figura 1.
Para determinação da condutividade hidráulica foram realizados sete ensaios utilizando o permeâmetro de Guelph a uma profundidade de $15 \mathrm{~cm}$. Considerando o procedimento adotado na realização do ensaio, foi utilizada uma altura de carga constante de $3 \mathrm{~cm}$ no per- 
meâmetro durante o ensaio. A determinação da condutividade hidráulica saturada K foi realizada através da equação:

$$
K=\frac{C \cdot Q}{\left(2 \cdot \pi \cdot \Pi^{2}+\Pi \cdot a^{2} \cdot C+\frac{2 \cdot \pi \cdot \pi}{\alpha}\right)}
$$

Onde: Ké a condutividade hidráulica saturada de campo, Q é vazão a carga constante, C é o fator de forma que depende da razão H/a e a é uma constante que depende das propriedades do solo.

A Tabela 3 apresenta os valores da condutividade hidráulica saturada obtidos para cada ponto através do ensaio com o permeâmetro de Guelph.

\section{Ensaios de laboratório para determinação da condutividade hidráulica}

Em função de o rejeito estudado apresentar características granulométricas típicas de areias médias e finas, a moldagem dos corpos-de-prova nas porosidades escolhidas foi realizada através de uma metodologia de reconstituição de amostras baseada na determinação da massa de material seco suficiente para preencher o permeâmetro de parede rígida, com 7,20 cm de diâmetro e 19,55 cm de altura, e, assim, atingir a porosidade requerida.

As técnicas de moldagem dos corpos-de-prova variaram em função da densidade adotada na remoldagem. Para os corpos-de-prova densos, foi utilizada a técnica de compactação dinâmica em material seco. Para os corpos-de-prova fofos foi utilizada a técnica de pluviação a seco. Finalmente, a compactação através de golpes na parede lateral do molde foi utilizada para obtenção dos corpos-de-prova com porosidades intermediárias (Presotti, 2002).

Na Tabela 4, estão representados os valores da condutividade hidráulica saturada determinada em laboratório para as amostras obtidas através do ESDH.

Os valores da condutividade hidráulica determinados em laboratório através de ensaios de carga constante utilizando as mesmas metodologias para as amostras de campo são mostrados na Tabela 5 .

Tabela 3 - Valores da condutividade hidráulica obtidos no campo com o permeâmetro de Guelph.

\begin{tabular}{c|c|c|c}
\hline Amostra & Fe (\%) & e & k (cm/s) \\
\hline Furo 01 & 21,64 & 0,79 & $7,68 \times 10^{-3}$ \\
\hline Furo 02 & 22,48 & 0,86 & $1,05 \times 10^{-3}$ \\
\hline Furo 03 & 21,44 & 0,84 & $1,05 \times 10^{-3}$ \\
\hline Furo 04 & 16,56 & 0,82 & $9,56 \times 10^{-3}$ \\
\hline Furo 05 & 23,72 & 0,80 & $9,07 \times 10^{-3}$ \\
\hline Furo 06 & 13,96 & 0,80 & $1,05 \times 10^{-2}$ \\
\hline Furo 07 & 13,88 & 0,82 & $1,10 \times 10^{-2}$ \\
\hline
\end{tabular}

\section{Análise dos resultados}

Objetivando obter a influência conjunta da porcentagem de ferro e da densidade na permeabilidade, o teor de ferro foi relacionado com a condutividade hidráulica. Assim, os resultados dos ensaios de carga constante foram agrupados considerando o estado de densidade e o teor de ferro, como mostra a Figura 2.

Analisando a Figura 2, pode-se verificar a influência da porcentagem de partículas de ferro presente nas amostras, onde o aumento do teor de ferro gerou uma redução no valor da condutividade hidráulica desse rejeito. Contudo essa influência mostrou-se um pouco mais evidente nas amostras mais fofas e tendendo a diminuir com a diminuição da porosidade inicial.

Ressalta-se que essa tendência de variação refere-se, especificamente, ao rejeito estudado e qualquer tipo de extrapolação para outros tipos de rejeitos de minérios de ferro seria prematuro, em vista das particularidades desses materiais. Contudo pode-se observar, de for-

Tabela 4 - Valores da condutividade hidráulica no ensaio de carga constante para as amostras do ESDH.

\begin{tabular}{c|c|c}
\hline Amostra & $\mathbf{e}$ & $\mathbf{k}(\mathbf{c m} / \mathbf{s})$ \\
\hline \multirow{4}{*}{ MA8-000 } & 0,67 & $8,37 \times 10^{-4}$ \\
\cline { 2 - 3 } & 0,81 & $1,75 \times 10^{-3}$ \\
\cline { 2 - 3 } & 0,98 & $2,09 \times 10^{-3}$ \\
\hline \multirow{4}{*}{ MA8-040 } & 0,66 & $4,68 \times 10^{-3}$ \\
\cline { 2 - 3 } & 0,77 & $7,82 \times 10^{-3}$ \\
\cline { 2 - 3 } & 0,91 & $9,90 \times 10^{-3}$ \\
\hline \multirow{4}{*}{ MA8-080 } & 0,62 & $5,92 \times 10^{-3}$ \\
\cline { 2 - 3 } & 0,70 & $8,71 \times 10^{-3}$ \\
\cline { 2 - 3 } & 0,86 & $1,12 \times 10^{-2}$ \\
\hline \multirow{4}{*}{ MA8-120 } & 0,62 & $7,66 \times 10^{-3}$ \\
\cline { 2 - 3 } & 0,70 & $1,03 \times 10^{-2}$ \\
\cline { 2 - 3 } & 0,86 & $1,54 \times 10^{-2}$ \\
\hline
\end{tabular}


Alexandre Gonçalves Santos et al.

ma clara, o efeito das propriedades dos rejeitos e do processo de deposição sobre a condutividade desses materiais.

Para os resultados de campo, foram realizadas as mesmas análises. A Figura 3 apresenta a variação da condutividade hidráulica obtida a partir dos ensaios de infiltração utilizando o permeâmetro de Guelph para os furos 1, 4 e 7. Observa-se a mesma tendência de redução da condutividade hidráulica com o aumento do teor de ferro. Contudo as diferenças obtidas foram menos significativas em vista da maior homogeneidade das amostras.

É importante ressaltar a influência do teor de ferro na condutividade hidráulica dos rejeitos, contudo a granulometria também apresenta uma importância fundamental. Caso fossem realizadas análises em regiões mais distantes do ponto de descarga, onde o teor de ferro é bastante baixo, a permeabilidade estaria relacionada, basicamente, à granulometria fina das partículas, que, certamente, se encontram nessas regiões.

\section{Conclusões}

A região do "Quadrilátero Ferrífero”, em Minas Gerais, é caracterizada pela presença de grandes complexos mineradores. A disposição dos resíduos gerados no processo de beneficiamento dessas minas tem sido normalmente realizada de forma aleatória e sem controle regular de operação.

Dessa forma, é importante notar que, para estudar o comportamento mecânico do rejeito granular, que é a fração utilizada na construção da barragem, além das características específicas desses materiais deve ser simulado o comportamento do rejeito após o processo de deposição.

Na caracterização do rejeito de minério de ferro, pode-se, facilmente, notar a influência do teor de ferro nas suas propriedades geotécnicas através das Tabelas 1 e 2. Com relação à densidade dos grãos, observou-se uma dependência direta desse parâmetro com o teor de
Tabela 5 - Valores da condutividade hidráulica no ensaio de carga constante para as amostras de campo.

\begin{tabular}{c|c|c|c}
\hline Amostra & Fe (\%) & e & $\mathbf{k}(\mathbf{c m} / \mathbf{s})$ \\
\hline Furo 01 & 21,64 & 0,79 & $8,54 \times 10^{-3}$ \\
\hline Furo 02 & 16,56 & 0,82 & $1,16 \times 10^{-2}$ \\
\hline Furo 03 & 21,44 & 0,84 & $1,09 \times 10^{-2}$ \\
\hline Furo 04 & 22,48 & 0,86 & $1,17 \times 10^{-2}$ \\
\hline Furo 05 & 23,72 & 0,80 & $1,17 \times 10^{-2}$ \\
\hline Furo 06 & 13,96 & 0,80 & $1,21 \times 10^{-2}$ \\
\hline Furo 07 & 13,88 & 0,82 & $1,23 \times 10^{-2}$ \\
\hline
\end{tabular}

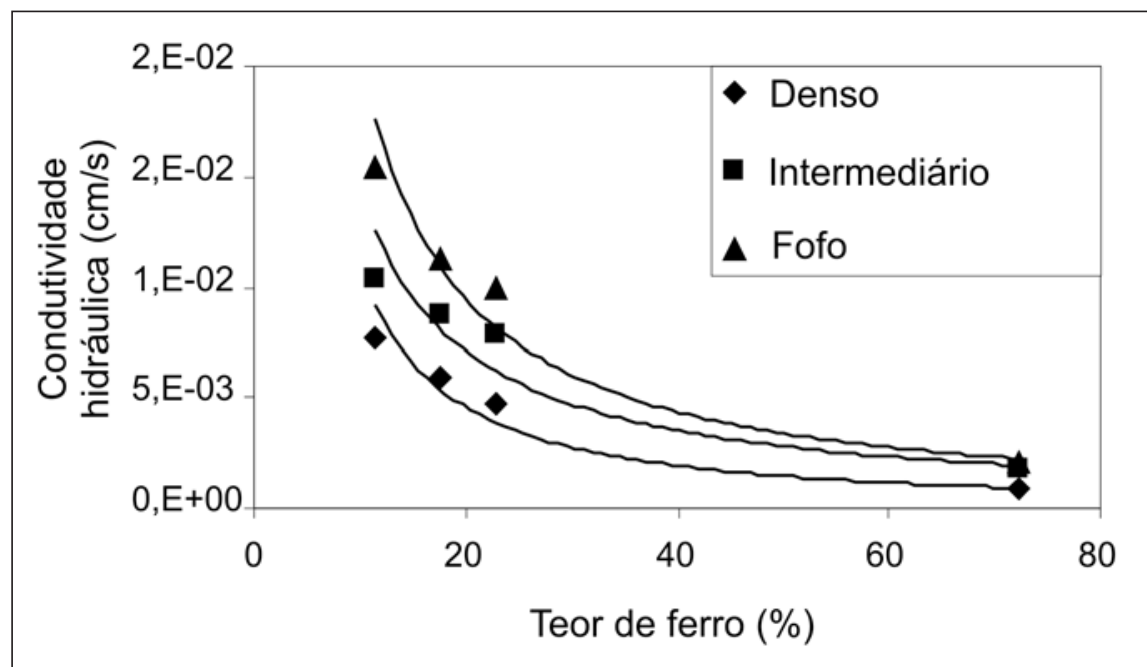

Figura 2 - Tendência de correlação entre o teor de ferro e a condutividade hidráulica das amostras obtidas no ESDH.

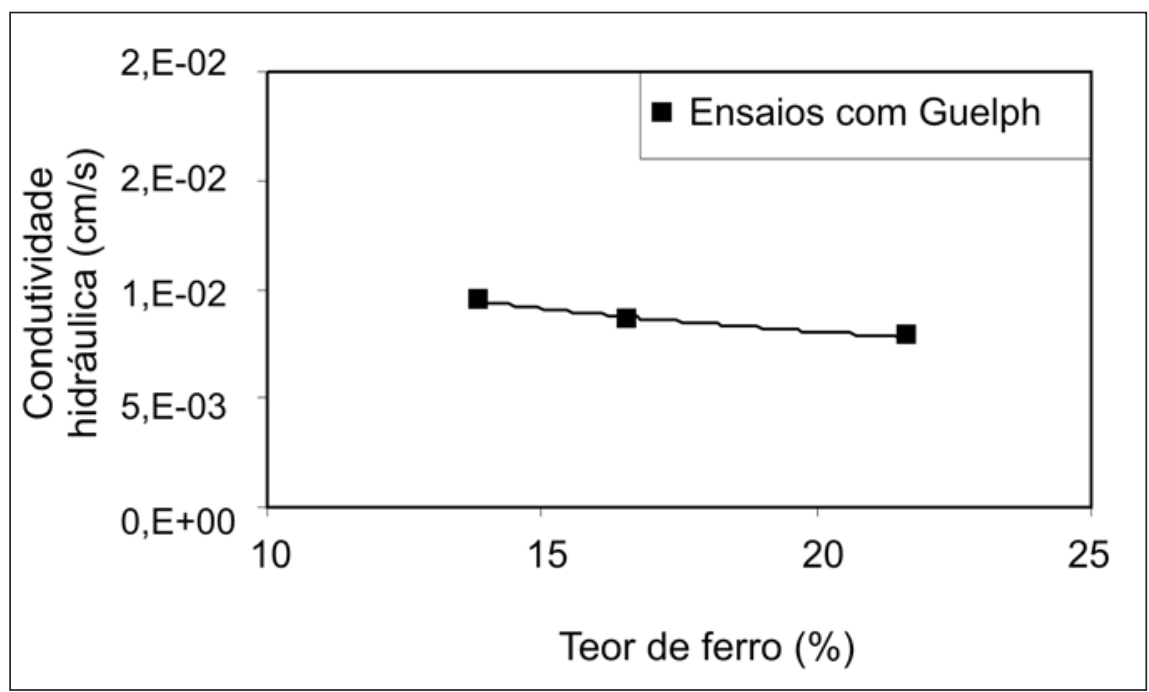

Figura 3 - Tendência de correlação entre o teor de ferro e a condutividade hidráulica das amostras obtidas no campo na seção 1-4-7. 
ferro. Assim, um aumento no teor de ferro tendo a provocar uma elevação considerável no valor da densidades dos grãos.

Nas análises da permeabilidade, o efeito do teor de ferro foi significativo para esse rejeito. As amostras com maiores teores de ferro apresentaram menores valores da condutividade hidráulica saturada. Verificou-se, ainda, que esse tipo de comportamento é mais significativo para estados de compacidade menores.

\section{Agradecimentos}

Esse trabalho é parte integrante de um amplo projeto de caracterização tecnológica de rejeitos de mineração, de- senvolvido pelo Núcleo de Geotecnia Aplicada à Mineração da Universidade Federal de Ouro Preto. Os autores gostariam de agradecer à Coordenação de Aperfeiçoamento de Pessoal de Nível Superior (CAPES) pelo apoio estrutural, à Companhia Vale do Rio Doce e à Universidade Federal de Ouro Preto.

\section{Referências bibliográficas}

ESPÓSITO, T.J. Metodologia probabilística e observacional aplicada a barragens de rejeito construídas por aterro hidráulico. Brasília: Universidade Federal de Brasília. 2000. (Tese de Doutorado).

PRESOTTI, E.S. Influência do teor de ferro nos parâmetros de resistência de um rejeito de minério de ferro. Ouro Preto: Universidade Federal de Ouro Preto, UFOP, 2002. 153p. (Dissertação de Mestrado).

RIBEIRO, L.F.M. Simulação física do processo de formação dos aterros hidráulicos aplicado a barragens de rejeitos. Brasília: Universidade Federal de Brasília. 2000. (Tese de Doutorado).

SOTO, M.A.A. Estudo da condutividade hidráulica em solos não saturados. São Paulo: Escola de Engenharia de São Carlos da Universidade Federal de São Paulo, 1999. (Dissertação de Mestrado).

VICK, S.G. Planning, design and analysis of tailings dams. New York: Wiley International, 1983.

Artigo recebido em 03/07/2006 e aprovado em 16/02/2007.

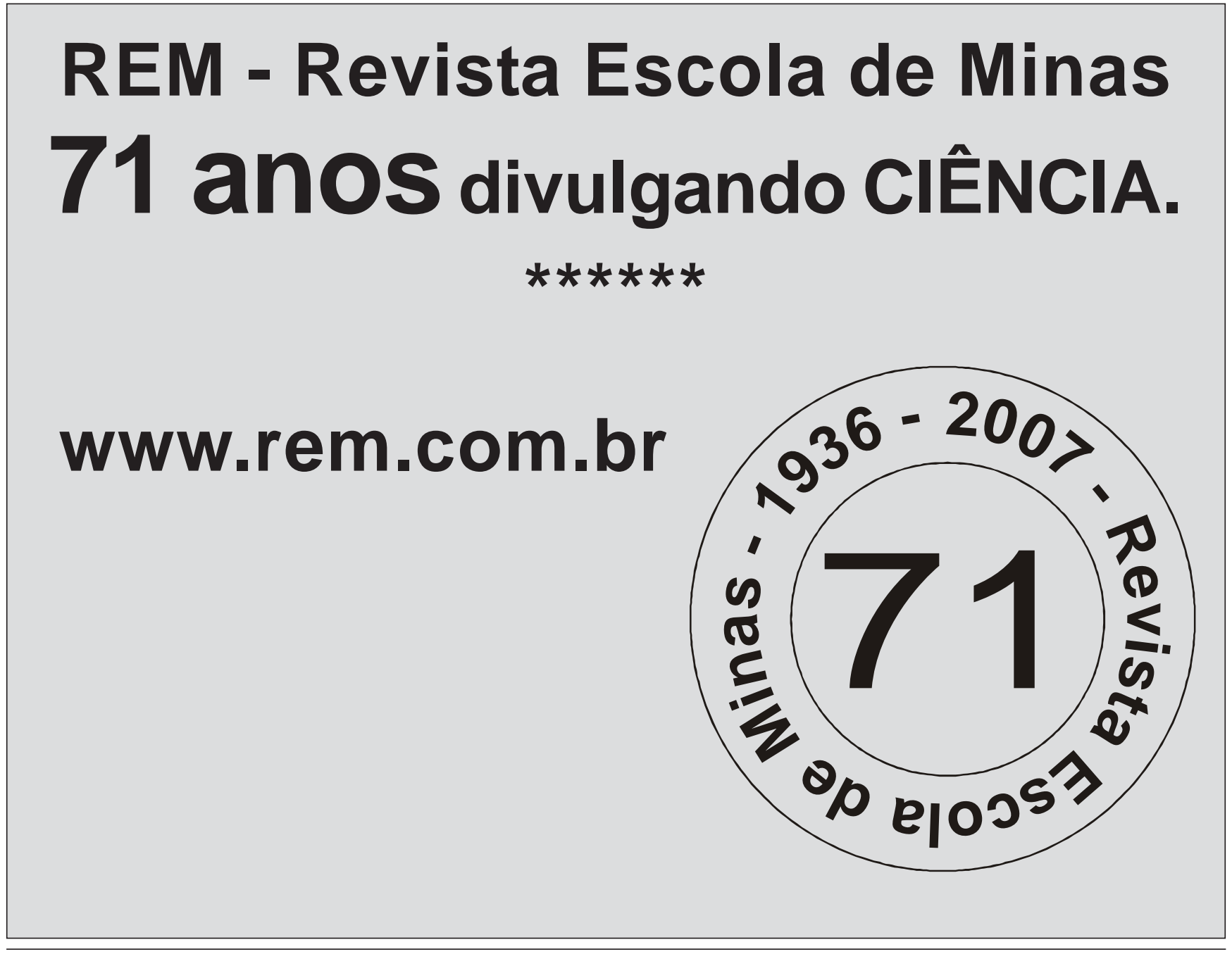

